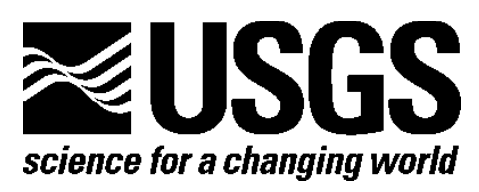

\title{
Noble Gas Isotopes in Mineral Springs within the Cascadia Forearc, Washington and Oregon
}

By Patricia A. McCrory, James E. Constantz, and Andrew G. Hunt

Open-File Report 2014-1064

U.S. Department of the Interior

U.S. Geological Survey 


\section{U.S. Department of the Interior \\ SALLY JEWELL, Secretary}

\section{U.S. Geological Survey \\ Suzette M. Kimball, Acting Director}

U.S. Geological Survey, Reston, Virginia: 2014

For more information on the USGS-the Federal source for science about the Earth, its natural and living resources, natural hazards, and the environment-visit http://www.usgs.gov or call 1-888-ASK-USGS

For an overview of USGS information products, including maps, imagery, and publications, visit http://www.usgs.gov/pubprod

To order this and other USGS information products, visit http://store.usgs.gov

Suggested citation:

McCrory, P.A., Constantz, J.E., and Hunt, A.G., 2014, Noble gas isotopes in mineral springs within the Cascadia Forearc, Wasihington and Oregon: U.S. Geological Survey Open-File Report 2014-1064, 20 p., http://dx.doi.org/10.3133/ofr20141064.

ISSN 2331-1258 (online)

Any use of trade, product, or firm names is for descriptive purposes only and does not imply endorsement by the U.S. Government.

Although this report is in the public domain, permission must be secured from the individual copyright owners to reproduce any copyrighted material contained within this report. 


\section{Contents}

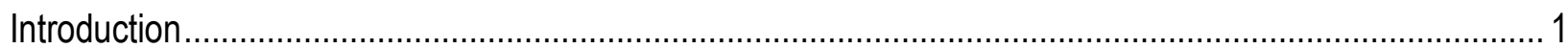

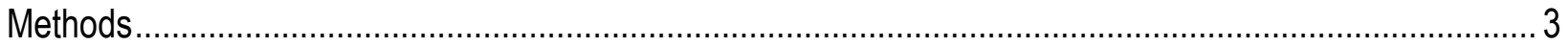

Field

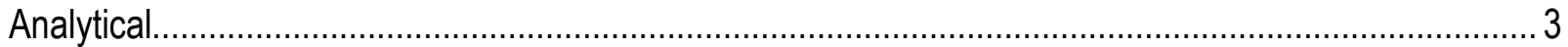

Mineral Spring Sites Sampled for Noble Gas Isotopes ....................................................................... 4

Sol Duc Mineral Springs, Olympic National Park, Washington ........................................................... 4

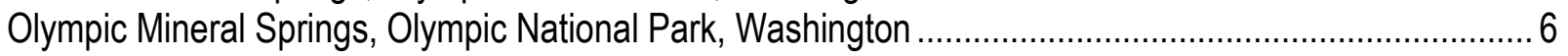

Well \#67, Jackson Prairie Natural Gas Storage Facility, Washington ................................................ 8

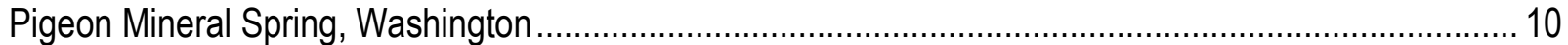

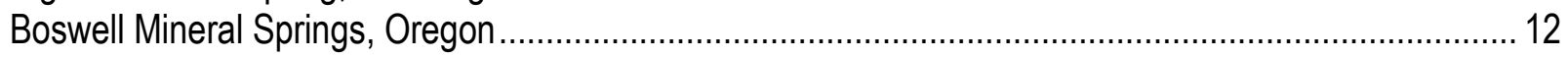

Cascadia Soda Spring, Cascadia State Park, Oregon .................................................................... 14

Sodaville Mineral Spring, Sodaville Park, Oregon............................................................................. 16

Wilhoit Soda Spring, Wilhoit County Park, Oregon ............................................................................... 18

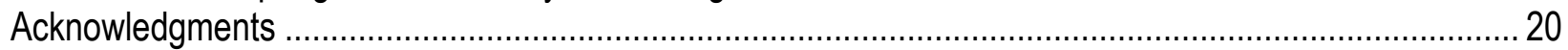

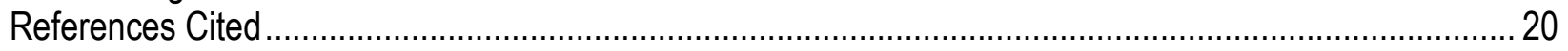

\section{Figure}

Figure 1. Map showing location of mineral spring sites with respect to Juan de Fuca plate model of McCrory and others (2012) and the location of the forearc mantle corner (McCrory and others, 2014) ........ 2 


\section{Conversion Factors and Datums}

\section{Conversion Factors}

Inch/Pound to SI

\begin{tabular}{lll}
\hline \multicolumn{1}{c}{ Multiply } & \multicolumn{1}{c}{ By } & \multicolumn{1}{c}{ To obtain } \\
\hline inch (in.) & Length & \\
foot (ft) & 2.54 & centimeter (cm) \\
\hline & 0.3048 & meter (m) \\
\hline pound, avoirdupois (lb) & Mass & \\
\hline
\end{tabular}

SI to Inch/Pound

\begin{tabular}{|c|c|c|}
\hline Multiply & By & To obtain \\
\hline \multicolumn{3}{|c|}{ Length } \\
\hline meter (m) & 3.281 & foot $(\mathrm{ft})$ \\
\hline kilometer $(\mathrm{km})$ & 0.6214 & mile (mi) \\
\hline \multicolumn{3}{|c|}{ Volume } \\
\hline liter (L) & 0.2642 & gallon (gal) \\
\hline
\end{tabular}

Temperature in degrees Celsius $\left({ }^{\circ} \mathrm{C}\right)$ may be converted to degrees Fahrenheit $\left({ }^{\circ} \mathrm{F}\right)$ as follows:

${ }^{\circ} \mathrm{F}=\left(1.8 x^{\circ} \mathrm{C}\right)+32$.

Specific conductance is given in microsiemens per centimeter at 25 degrees Celsius $\left(\mu \mathrm{S} / \mathrm{cm}\right.$ at $\left.25^{\circ} \mathrm{C}\right)$.

\section{Datums}

Horizontal coordinate information is referenced to the World Geodetic System of 1984 (WGS84).

Vertical coordinate information is referenced to the World Geodetic System Earth Gravitational Model Geoid of 1996 (WGS84 EGM96 Geoid).

Elevation, as used in this report, refers to distance above mean sea level. 


\title{
Noble Gas Isotopes in Mineral Springs within the Cascadia Forearc, Washington and Oregon
}

\author{
By Patricia A. McCrory, James E. Constantz, and Andrew G. Hunt
}

\section{Introduction}

This U.S. Geological Survey report presents laboratory analyses along with field notes for a pilot study to document the relative abundance of noble gases in mineral springs within the Cascadia forearc of Washington and Oregon (fig. 1). Estimates of the depth to the underlying Juan de Fuca oceanic plate beneath the sample sites are derived from the McCrory and others (2012) slab model. Some of these springs have been previously sampled for chemical analyses (Mariner and others, 2006), but none currently have publicly available noble gas data. Helium isotope values as well as the noble gas values and ratios presented below will be used to determine the sources and mixing history of these mineral waters. 


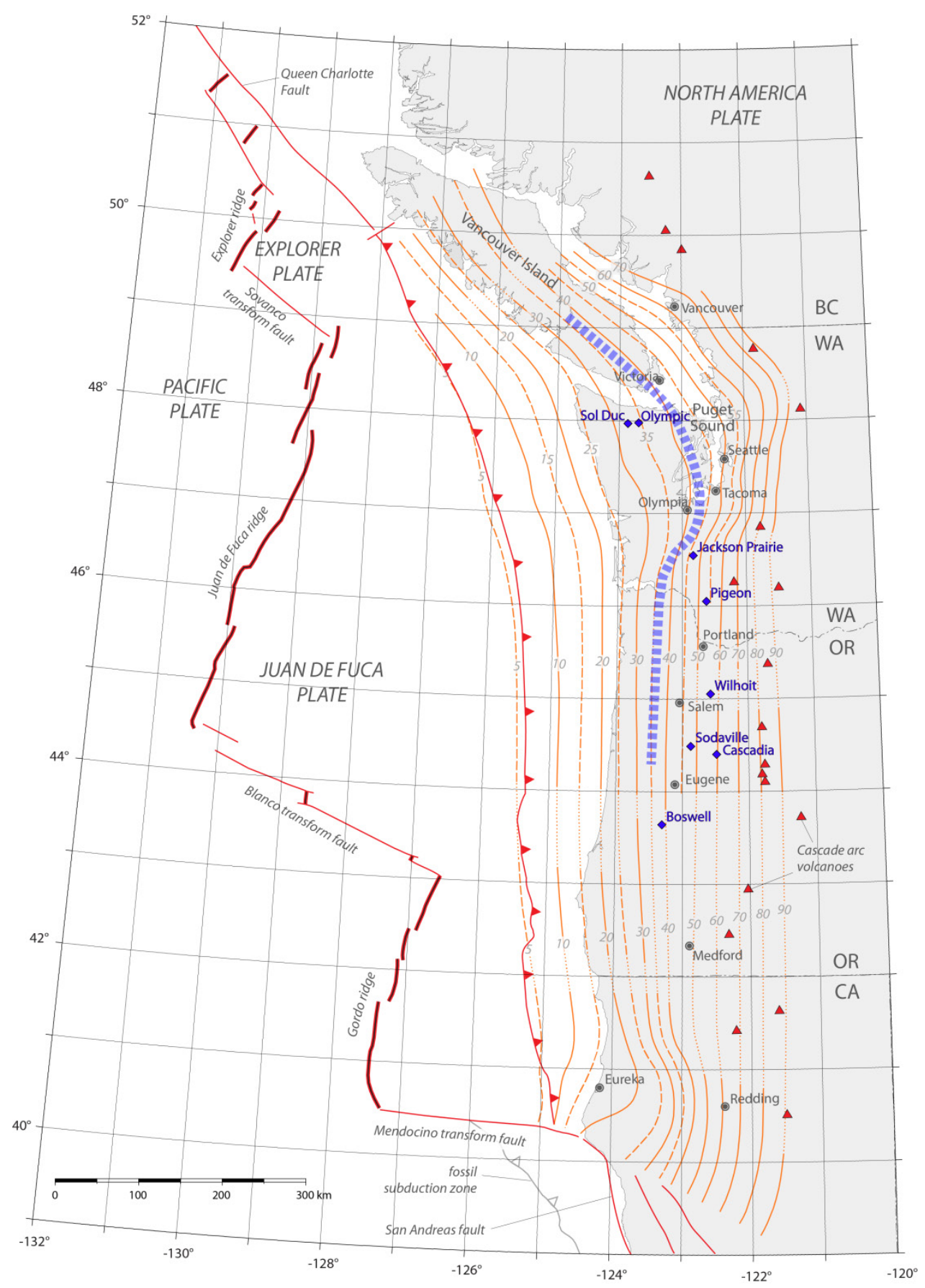

Figure 1. Map showing location of mineral spring sites with respect to Juan de Fuca plate model of McCrory and others (2012) and the location of the forearc mantle corner (McCrory and others, 2014). Iso-depth contours of the Juan de Fuca slab are in kilometers below surface. Thick dashed line denotes forearc mantle corner; labeled blue diamonds denote mineral spring sites; red triangles denote Cascade arc volcanoes. Transverse Mercator projection, WGS84 datum, standard parallel $-128^{\circ}$, centered at $46.8^{\circ} \mathrm{N},-128^{\circ} \mathrm{W}$, with standard parallel rotated $3^{\circ}$ clockwise of vertical (plate boundaries modified from Wilson, 2002). 


\section{Methods}

Field

Water samples were collected from the mineral and soda springs in copper tubes that are pinched shut at each end with metal clamps. We first established siphon flow through Tygon ${ }^{\circledR}$ tubing attached to both ends of an 18-in. length of copper tubing. The copper tube was tilted so that water flowed vertically upward during collection. We then gently tapped the outside of the copper tube just before tightening the downstream clamp to release any bubbles that might be stuck to the inside wall of the tube. The nuts on the downstream clamp were slowly tightened using a torque wrench set to $10 \mathrm{ft}-\mathrm{lbs}$. After the downstream clamp was closed, we closed the upstream clamp. Finally, we removed the Tygon ${ }^{\circledR}$ tubes, filled both ends of the copper tube with spring water, and sealed the ends with rubber caps.

Sample site locations and elevations were determined with a handheld GPS device that utilizes horizontal and vertical WGS84 datums.

\section{Analytical}

Gas analyses were performed in the U.S Geological Survey Noble Gas Laboratory in Denver, Colorado. Gas samples were released into an ultra-low vacuum extraction line $\left(<5 \times 10^{-9}\right.$ torr) and passed through a set of ethanol/dry ice slush (approximately $-72{ }^{\circ} \mathrm{C}$ ) traps to remove water vapor. The volume of dry gas was measured with a calibrated capacitance manometer and an aliquot was introduced to a quadrupole mass spectrometer for analysis of bulk gas (hydrogen, methane, nitrogen, and carbon dioxide). The remaining gas was exposed to a STS-101 getter heated to $350{ }^{\circ} \mathrm{C}$ to remove reactive-gas components, leaving a mixed sample of the noble gases. An aliquot of the purified gas was admitted to a MAP-215-50 magnetic-sector mass spectrometer for analysis of argon, krypton, and xenon $\left({ }^{36} \mathrm{Ar},{ }^{38} \mathrm{Ar},{ }^{40} \mathrm{Ar},{ }^{84} \mathrm{Kr},{ }^{86} \mathrm{Kr},{ }^{130} \mathrm{Xe}\right.$, and $\left.{ }^{132} \mathrm{Xe}\right)$, while the remaining gas was cryogenically separated and analyzed for both helium $\left({ }^{3} \mathrm{He}\right.$ and $\left.{ }^{4} \mathrm{He}\right)$ and neon isotopes $\left({ }^{20} \mathrm{Ne},{ }^{21} \mathrm{Ne}\right.$, and $\left.{ }^{22} \mathrm{Ne}\right)$. Gas from dissolved gas samples was separated using a gas extraction bulb connected to the ultra-low vacuum line. Each sample was introduced to the extraction bulb under vacuum and exposed to 15 minutes of agitation in an ultrasonic bath at 30 ${ }^{\circ} \mathrm{C}$ to promote complete extraction of dissolved gas. The extracted gas was then analyzed using the same method described above and presented as total elemental composition rather than isotopic composition. The resulting gas concentrations are based on the calibrated responses of the mass spectrometers and total volume of extracted gas. Noble gas solubilities were derived from Weiss (1970, 1971), Weiss and Kyser (1970), and Benson and Krause (1976). 


\section{Mineral Spring Sites Sampled for Noble Gas Isotopes}

\section{Sol Duc Mineral Springs, Olympic National Park, Washington}

\section{Bogachiel Peak 7.5-minute topographic quadrangle map. Sampled August 2012. Juan de}

Fuca slab depth approximately $33 \mathrm{~km}$.

[Microsoft ${ }^{\circledR}$ Excel file of this table is available for download at http://pubs.usgs.gov/of/2014/1064/]

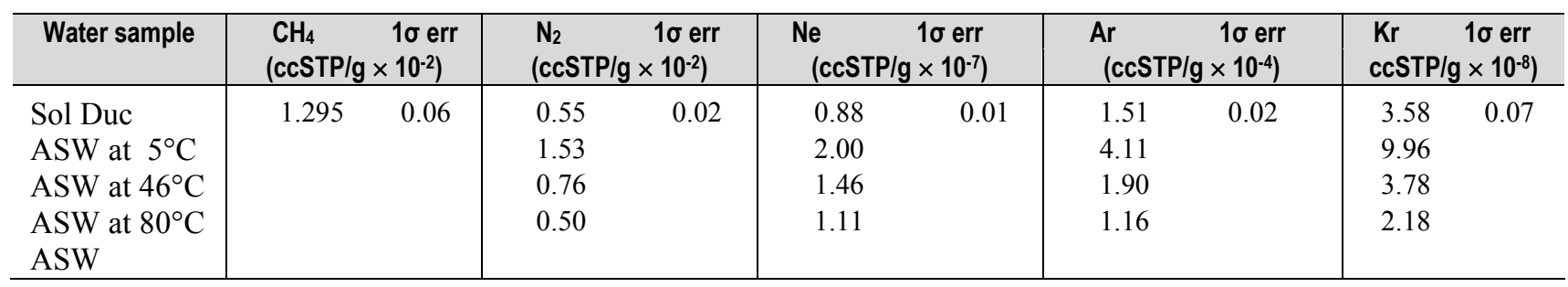

\begin{tabular}{|c|c|c|c|c|c|c|c|c|c|}
\hline Water sample & $\begin{array}{l}\mathrm{Xe} \quad 1 \sigma \mathrm{err} \\
\left(\mathrm{ccSTP} / \mathrm{g} \times 10^{-8}\right)\end{array}$ & ${ }^{20} \mathrm{Ne} /{ }^{22} \mathrm{Ne}$ & $1 \sigma \mathrm{err}$ & ${ }^{40} \mathrm{Ar} /{ }^{36} \mathrm{Ar}$ & $1 \sigma \mathrm{err}$ & ${ }^{86} \mathrm{Kr} /{ }^{84} \mathrm{Kr}$ & $1 \sigma$ err & ${ }^{130} \mathrm{Xe} /{ }^{132} \mathrm{Xe}$ & $1 \sigma$ err \\
\hline $\begin{array}{l}\text { Sol Duc } \\
\text { ASW at } 5^{\circ} \mathrm{C} \\
\text { ASW at } 46^{\circ} \mathrm{C} \\
\text { ASW at } 80^{\circ} \mathrm{C} \\
\text { ASW }\end{array}$ & $\begin{array}{ll}0.32 & 0.01 \\
1.49 & \\
0.46 & \\
0.17 & \end{array}$ & 9.83 & 0.023 & 294.4 & 1.4 & 0.306 & 0.002 & 0.147 & 0.003 \\
\hline
\end{tabular}

Run notes:

1. ASW (Air Saturated Water) computed for listed temperatures at an elevation of 1,600 ft; no salinity correction was applied to the gas solubility data.

2. Samples do not calculate back to a single solubility temperature, represent a mix of colder and warmer water that has been heated, and is degassing at the pool interface. 
20120823-01 PMc (water)

$\mathrm{N} 47^{\circ} 58.125^{\prime} \mathrm{W} 123^{\circ} 51.793 '$

Elevation: $1,629 \mathrm{ft}$

Water temperature: approximately $49.8^{\circ} \mathrm{C}$

Specific conductance: $334 \mu \mathrm{S} / \mathrm{cm}$

The main mineral spring at this site feeds commercially run soaking pools within the Olympic National Park.

The operations manager of Sol Duc Hot Springs Resort opened the wooden cover to the mineral spring source. The water level was about $10 \mathrm{ft}$ below deck level, however, too deep for siphon sampling. Instead, the water sample was drawn from a hose connected to the spring (after letting water run from hose for about 5 minutes). Sample collected using siphon feed.

This mineral spring has been previously sampled for chemical analyses by Roland Tabor (June 1970), and by Robert Mariner and William Evans (July 1977) as reported in Mariner and others (2006). 


\section{Olympic Mineral Springs, Olympic National Park, Washington}

Mount Carrie 7.5-minute topographic quadrangle map. Sampled August 2012. Juan de Fuca slab depth approximately $35 \mathrm{~km}$.

[Microsoft ${ }^{\circledR}$ Excel file of this table is available for download at http://pubs.usgs.gov/of/2014/1064]

\begin{tabular}{|c|c|c|c|c|c|}
\hline Water sample & $\begin{array}{l}\mathrm{CH}_{4} \quad 1 \sigma \mathrm{err} \\
\left(\mathrm{ccSTP} / \mathrm{g} \times 10^{-2}\right)\end{array}$ & $\begin{array}{c}\mathrm{N}_{2} \quad 1 \sigma \mathrm{err} \\
\left(\mathrm{ccSTP} / \mathrm{g} \times 10^{-2}\right)\end{array}$ & $\begin{array}{l}\mathrm{Ne} \\
\left(\mathrm{ccSTP} / \mathrm{g} \times 10^{-7}\right)\end{array}$ & $\begin{array}{l}\text { Ar } \quad 1 \sigma \text { err } \\
\left(\mathrm{ccSTP} / g \times 10^{-4}\right)\end{array}$ & $\begin{array}{l}\mathrm{Kr} \quad 1 \sigma \mathrm{err} \\
\left(\mathrm{ccSTP} / \mathrm{g} \times 10^{-8}\right)\end{array}$ \\
\hline $\begin{array}{l}\text { Olympic \#4 } \\
\text { ASW at } 5^{\circ} \mathrm{C}\end{array}$ & $0.860 \quad 0.04$ & $\begin{array}{ll}0.66 & 0.02 \\
1.53 & \end{array}$ & $\begin{array}{ll}0.91 & 0.01 \\
2.00 & \end{array}$ & $\begin{array}{l}2.16 \\
4.11\end{array}$ & $\begin{array}{ll}5.17 & 0.1 \\
9.96 & \end{array}$ \\
\hline $\mathrm{ASW}$ at $46^{\circ} \mathrm{C}$ & & 0.76 & 1.46 & 1.90 & 3.78 \\
\hline $\begin{array}{l}\mathrm{ASW} \text { at } 80^{\circ} \mathrm{C} \\
\mathrm{ASW}\end{array}$ & & 0.50 & 1.11 & 1.16 & 2.18 \\
\hline
\end{tabular}

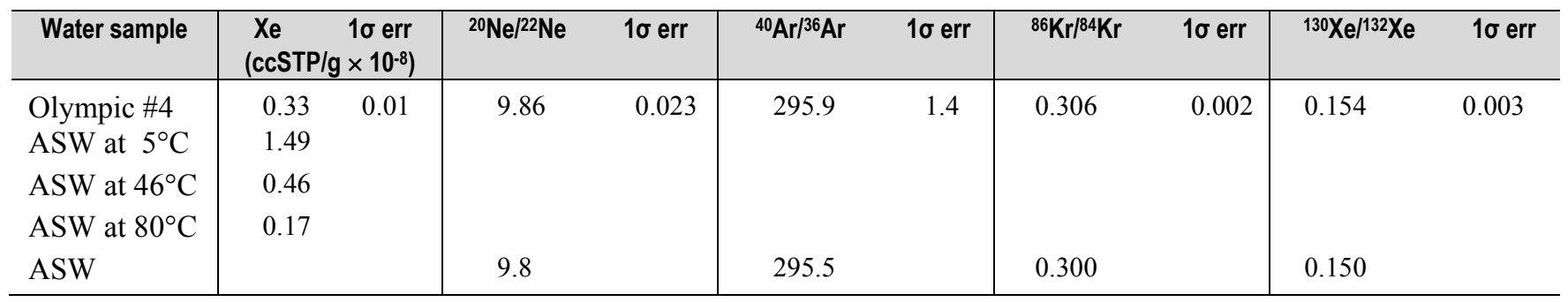

Run notes:

1. ASW (Air Saturated Water) computed for listed temperatures at an elevation of 1,600 ft; no salinity correction was applied to the gas solubility data.

2. Samples do not calculate back to a single solubility temperature, represent a mix of colder and warmer water that has been heated, and is degassing at the pool interface. 
20120824-01 PMc (water)

$\mathrm{N} 47^{\circ} 58.582{ }^{\prime} \mathrm{W} 123^{\circ} 41.3039 ' \pm 19 \mathrm{ft}$

Elevation: $2,177 \pm 4 \mathbf{f t}$

Water temperature: approximately $45.8^{\circ} \mathrm{C}$

Specific conductance: $290 \mu \mathrm{S} / \mathrm{cm}$

A series of at least six mineral springs seep from the hillside along a trail within Olympic National Park. Public road access to the trailhead was closed in 2012 because of ongoing dam removal on the upper Elwah River (otherwise hikers would likely have been soaking in the hot springs). A park ranger unlocked the gate above the ranger station and arranged for road access past the dam removal site to the trailhead. The springs are located about a 45 -minute hike from the trailhead.

Water temperature and specific conductance were measured for each of six springs, and the hottest spring was sampled. This spring also had the most gas bubbles venting from bottom of its pool. Our initial attempt to sample gas bubbles proved to be unsuccessful as too few bubbles discharged from the bottom of the pool, and from variable spots at irregular intervals. Sample collected using siphon feed.

This mineral spring has been previously sampled for chemical analyses by Roland Tabor (June 1969), and by Robert Mariner and William Evans (July 1977) as reported in Mariner and others (2006).

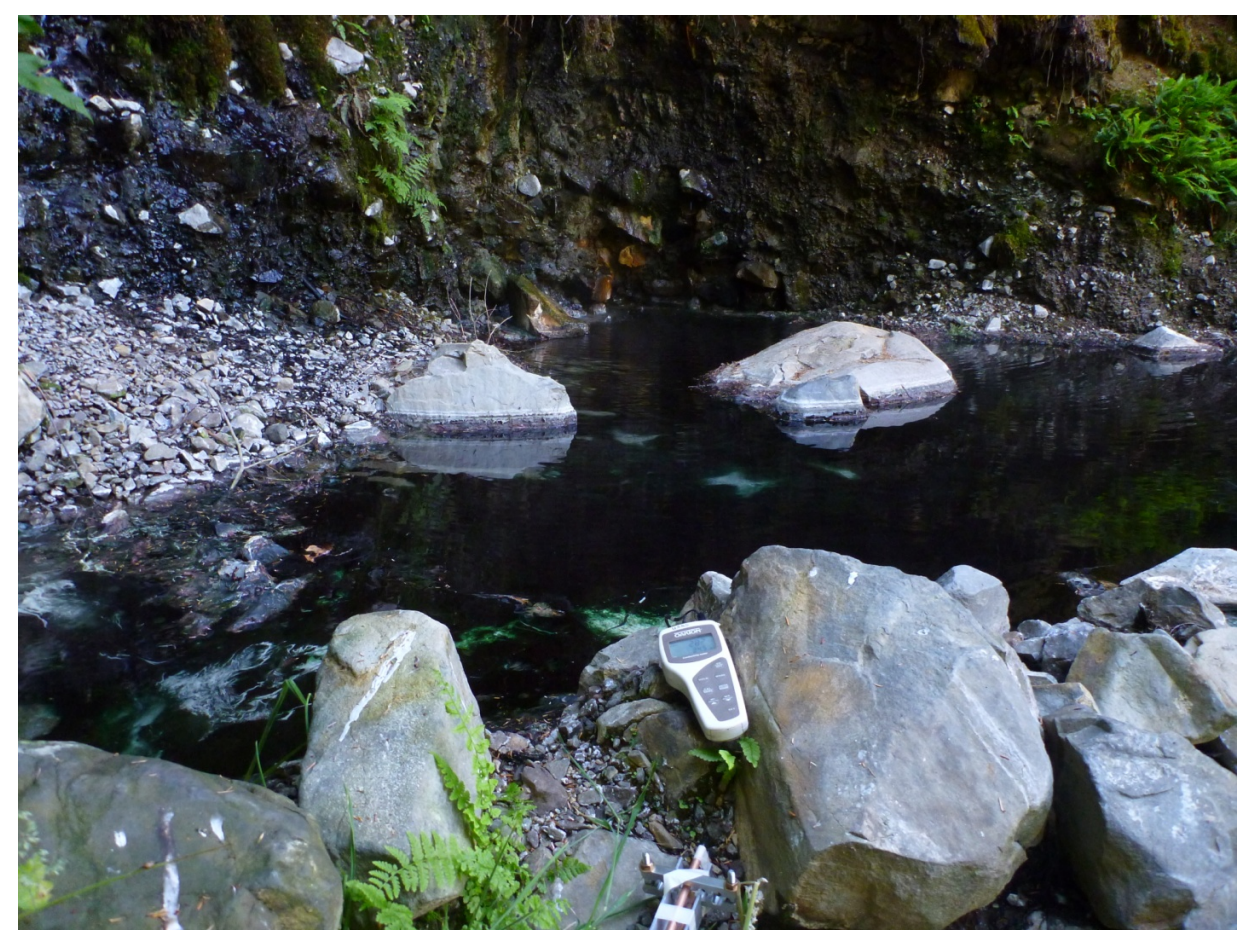




\section{Well \#67, Jackson Prairie Natural Gas Storage Facility, Washington}

Jackson Prairie 7.5-minute topographic quadrangle map. Sampled August 2012. Juan de Fuca slab depth approximately $47 \mathrm{~km}$.

[Microsoft ${ }^{\mathbb{B}}$ Excel file of this table is available for download at http://pubs.usgs.gov/of/2014/1064/]

\begin{tabular}{|c|c|c|c|c|}
\hline Water sample & $\begin{array}{l}\mathrm{CH}_{4} \quad 1 \sigma \mathrm{err} \\
\left(\mathrm{ccSTP} / \mathrm{g} \times 10^{-2}\right) \\
\end{array}$ & $\begin{array}{lc}\mathrm{N}_{2} & 1 \sigma \mathrm{err} \\
\left(\mathrm{ccSTP} / \mathrm{g} \times 10^{-2}\right) \\
\end{array}$ & $\begin{array}{l}\mathrm{Ne} \quad 1 \sigma \mathrm{err} \\
\left(\mathrm{ccSTP} / \mathrm{g} \times 10^{-7}\right) \\
\end{array}$ & $\begin{array}{l}\mathrm{Ar} \quad 1 \sigma \mathrm{err} \\
\left(\mathrm{ccSTP} / \mathrm{g} \times 10^{-4}\right)\end{array}$ \\
\hline $\begin{array}{l}\text { Mary's Corner } \\
\text { ASW at } 20^{\circ} \mathrm{C}\end{array}$ & 1.917 & $\begin{array}{l}0.6 \\
1.17\end{array}$ & $\begin{array}{l}0.518 \\
18.17\end{array}$ & $\begin{array}{l}0.89 \\
3.06\end{array}$ \\
\hline
\end{tabular}

\begin{tabular}{c|l|l|l|ll}
\hline Water sample & $\begin{array}{l}\mathrm{Kr} 1 \sigma \mathrm{err} \\
\left.\mathrm{ccSTP} / \mathrm{g} \times 10^{-8}\right)\end{array}$ & $\begin{array}{l}\mathrm{Xe} \quad 1 \sigma \mathrm{err} \\
\left.\mathrm{ccSTP} / \mathrm{g} \times 10^{-8}\right)\end{array}$ & ${ }^{20} \mathrm{Ne} / 22 \mathrm{Ne} \quad 1 \sigma \mathrm{err}$ & ${ }^{21} \mathrm{Ne} / 22 \mathrm{Ne}$ & $1 \sigma$ err \\
\hline Mary's Corner & 2.0 & 0.28 & 9.841 & 0.028 \\
ASW at $20^{\circ} \mathrm{C}$ & 6.84 & 0.93 & & \\
\hline
\end{tabular}

\begin{tabular}{c|cc|cc|cc|cc}
\hline Water sample & ${ }^{38} \mathrm{Ar} /{ }^{36} \mathrm{Ar}$ & $1 \sigma \mathrm{err}$ & ${ }^{40} \mathrm{Ar} /{ }^{36} \mathrm{Ar}$ & $1 \sigma \mathrm{err}$ & ${ }^{86} \mathrm{Kr} /{ }^{84} \mathrm{Kr}$ & $1 \sigma \mathrm{err}$ & ${ }^{130} \mathrm{Xe} /{ }^{132} \mathrm{Xe}$ & $1 \sigma \mathrm{err}$ \\
\hline $\begin{array}{l}\text { Mary's Corner } \\
\text { ASW at } 20^{\circ} \mathrm{C}\end{array}$ & 0.216 & & 299.4 & & 0.304 & & 0.154 \\
\hline
\end{tabular}

\begin{tabular}{|c|c|c|c|c|}
\hline Gas sample & $\begin{array}{l}\mathrm{H}_{2} \quad 1 \sigma \mathrm{err} \\
\text { (ccSTP/cc) }\end{array}$ & $\begin{array}{c}\mathrm{CH}_{4} \quad 1 \sigma \mathrm{err} \\
\text { (ccSTP/cc) }\end{array}$ & $\begin{array}{c}\mathrm{N}_{2} \quad 1 \sigma \text { err } \\
\text { (ccSTP/cc) }\end{array}$ & $\begin{array}{c}\mathrm{C}_{2} \mathrm{H}_{6} \quad 1 \sigma \mathrm{err} \\
\text { (ccSTP/c) }\end{array}$ \\
\hline Mary's Corner & $<0.002$ & 0.583 & 0.414 & $<0.002$ \\
\hline
\end{tabular}

\begin{tabular}{|c|c|c|c|c|}
\hline Gas sample & $\begin{array}{cr}\mathrm{O}_{2} & 1 \sigma \mathrm{err} \\
& \text { (ccSTP/cc) }\end{array}$ & $\begin{array}{c}\mathrm{CO}_{2} \quad 1 \sigma \text { err } \\
\text { ccSTP/cc) }\end{array}$ & $1 \sigma$ err & $1 \sigma$ err \\
\hline Mary's Corner & $<0.003$ & $<0.002$ & 28 & 227 \\
\hline
\end{tabular}

\begin{tabular}{|c|c|c|c|c|c|c|c|c|}
\hline Gas sample & ${ }^{84} \mathrm{Kr}$ & $1 \sigma \mathrm{err}$ & ${ }^{132} \mathrm{Xe}$ & $1 \sigma$ err & ${ }^{20} \mathrm{Ne} / 22 \mathrm{Ne}$ & $1 \sigma \mathrm{err}$ & ${ }^{21} \mathrm{Ne} /{ }^{22} \mathrm{Ne}$ & $1 \sigma \mathrm{err}$ \\
\hline Mary's Corner & 19.98 & & 1.22 & & 9.698 & & 0.0304 & \\
\hline Gas sample & ${ }^{38} \mathrm{Ar} /{ }^{36} \mathrm{Ar}$ & $1 \sigma \mathrm{err}$ & ${ }^{40} \mathrm{Ar} /{ }^{36} \mathrm{Ar}$ & $1 \sigma \mathrm{err}$ & ${ }^{86} \mathrm{Kr} /{ }^{84} \mathrm{Kr}$ & $1 \sigma \mathrm{err}$ & ${ }^{130} \mathrm{Xe} /{ }^{132} \mathrm{Xe}$ & $1 \sigma \mathrm{err}$ \\
\hline Mary's Corner & 0.182 & & 463.2 & & 0.299 & & 0.154 & \\
\hline
\end{tabular}

Run notes:

1. ASW (Air Saturated Water) computed for listed temperature at an elevation of $500 \mathrm{ft}$.

2. Gas sample 50 percent methane. Gas sample contains excess ${ }^{40} \mathrm{Ar}$; excess ${ }^{40} \mathrm{Ar}$ masked in water sample by the water. Gas sample contains a little excess ${ }^{20} \mathrm{Ne}$; excess Neon not seen in the water sample. Almost no $\mathrm{CO}_{2}$. N/Ar value not the best.

3. $1 \sigma$ error for noble gases is approximately $1-2$ percent. 
20120826-01 PMc (water)

$\mathrm{N} 46^{\circ} 32.711^{\prime} \mathrm{W} 122^{\circ} 49.474^{\prime}$

Elevation: $527 \mathrm{ft}$

Water temperature: approximately $32.2^{\circ} \mathrm{C}$

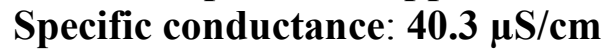

We initially met with the Jackson Prairie Natural Gas Storage Facility manager, then followed one of the reservoir operators to Well \#67. This well has a small storage tank on top. The reservoir operator opened the lower valve on the tank to release groundwater. He let the water flow for a few minutes to bleed pressure in the tank. Even so, the water sample contained a fair amount of gas bubbles. We then measured water temperature and specific conductance. The water and gas samples come from marine sandstone layers at a depth of approximately $600 \mathrm{~m}$ in the Chehalis sedimentary basin. Note, noble gas abundances reflect a mixture of local and distant sources as methane is piped from western Canada and Rocky Mountain sources, and injected into the Chehalis Basin for seasonal storage.

\section{6-02 PMc (gas)}

After collecting the water sample, the reservoir operator opened the upper nozzle on the storage tank to collect a gas sample. This required two attempts as the gas was under about $60 \mathrm{lbs}$ of pressure and blew the hose off the sampling device on the first attempt to tighten the downstream clamp.

This well has been previously sampled for chemical analyses by Ivan Barnes (September 1983), and by Rodney Caldwell and Steve Hinkle (1993) as reported in Mariner and others (2006).

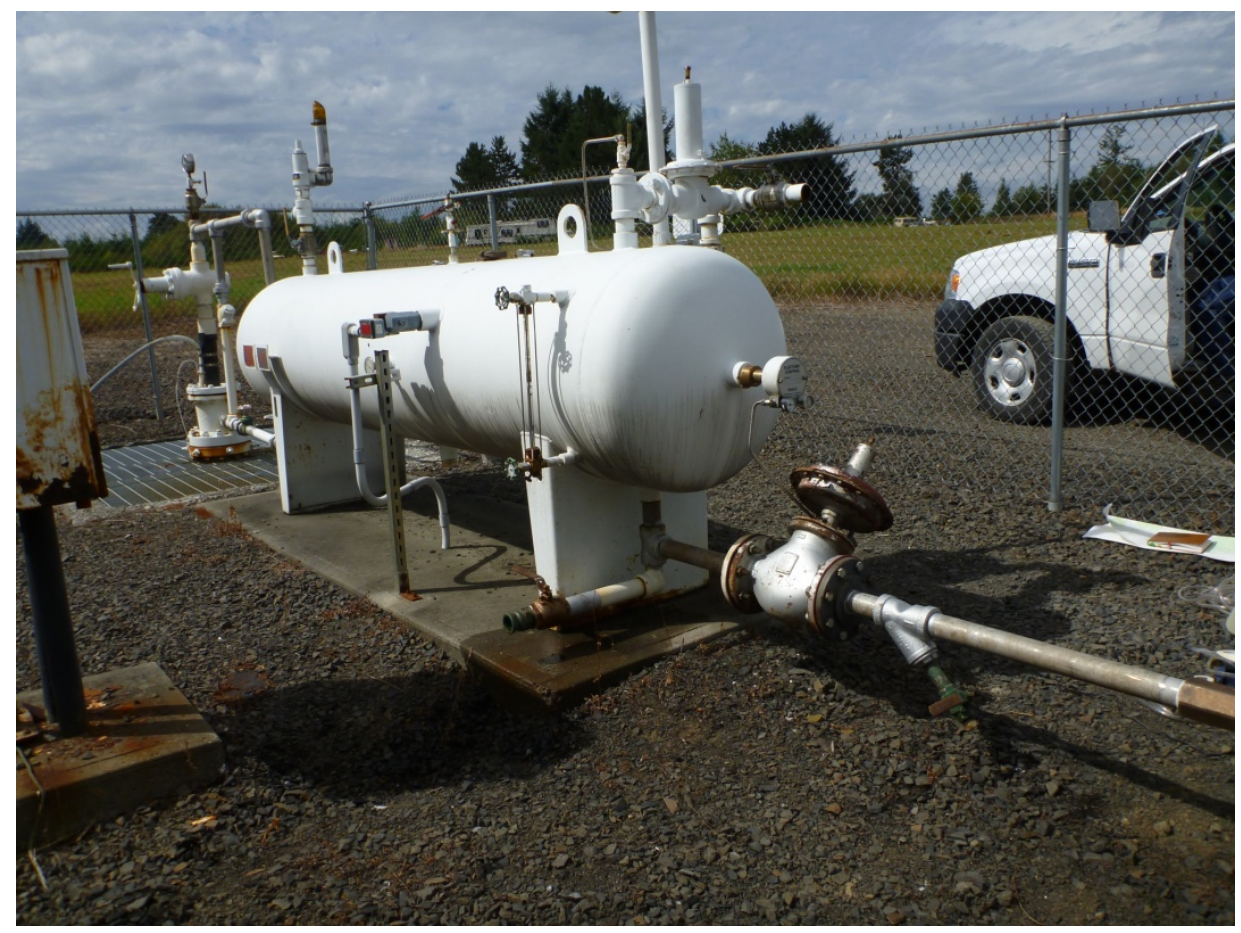


Pigeon Mineral Spring, Washington

Georges Peak 7.5-minute topographic quadrangle map. Sampled August 2013. Juan de Fuca slab depth approximately $53 \mathrm{~km}$.

[Microsoft ${ }^{\circledR}$ Excel file of this table is available for download at http://pubs.usgs.gov/of/2014/1064/]

\begin{tabular}{|c|c|c|c|c|}
\hline Water sample & $\begin{array}{l}\mathrm{CH}_{4} \quad 1 \sigma \mathrm{err} \\
\left(\mathrm{ccSTP} / \mathrm{g} \times 10^{-3}\right) \\
\end{array}$ & $\begin{array}{lc}\mathrm{N}_{2} & 1 \sigma \mathrm{err} \\
\left(\mathrm{ccSTP} / \mathrm{g} \times 10^{-2}\right) \\
\end{array}$ & $\begin{array}{lr}\mathrm{O}_{2} & 1 \sigma \mathrm{err} \\
\left(\mathrm{ccSTP} / \mathrm{g} \times 10^{-3}\right) \\
\end{array}$ & $\begin{array}{ll}\mathrm{Ne} & 1 \sigma \mathrm{er} \\
\left(\mathrm{ccSTP} / \mathrm{g} \times 10^{-8}\right)\end{array}$ \\
\hline Pigeon & $<0.02$ & 1.75 & $<0.02$ & 0.45 \\
\hline $\mathrm{ASW}$ at $20^{\circ} \mathrm{C}$ & & 1.17 & & 18.17 \\
\hline
\end{tabular}

\begin{tabular}{|c|c|c|c|c|c|}
\hline Water sample & $\begin{array}{l}\mathrm{Ar} \quad 1 \sigma \mathrm{err} \\
\left(\mathrm{ccSTP} / \mathrm{g} \times 10^{-4}\right) \\
\end{array}$ & $\begin{array}{c}\mathrm{Kr} \quad 1 \sigma \mathrm{err} \\
\left(\mathrm{ccSTP} / \mathrm{g} \times 10^{-8}\right)\end{array}$ & $\begin{array}{c}\mathrm{Xe} \quad 1 \sigma \mathrm{err} \\
\left(\mathrm{ccSTP} / \mathrm{g} \times 10^{-8}\right)\end{array}$ & ${ }^{20} \mathrm{Ne} / 22 \mathrm{Ne}$ & $1 \sigma \mathrm{err}$ \\
\hline Pigeon & 0.55 & 2.29 & 0.57 & 10.62 & \\
\hline $\mathrm{ASW}$ at $20^{\circ} \mathrm{C}$ & 0.306 & 6.84 & 0.93 & & \\
\hline
\end{tabular}

\begin{tabular}{l|cc|cc|cc}
\hline Water sample & ${ }^{40} \mathrm{Ar} / 36 \mathrm{Ar} \quad 1 \sigma$ err & ${ }^{86} \mathrm{Kr} /{ }^{84} \mathrm{Kr}$ & $1 \sigma \mathrm{err}$ & ${ }^{130} \mathrm{Xe} /{ }^{132} \mathrm{Xe} \quad 1 \sigma \mathrm{err}$ \\
\hline $\begin{array}{l}\text { Pigeon } \\
\text { ASW at } 20^{\circ} \mathrm{C}\end{array}$ & 378.6 & 0.306 & & 0.150 & \\
\hline
\end{tabular}

Run notes:

1. ASW (Air Saturated Water) computed for listed temperature at an elevation of $500 \mathrm{ft}$.

2. Only minor $\mathrm{CO}_{2}$ noted.

3. $1 \sigma$ error for noble gases is approximately $1-2$ percent. 
20130819-01 PMc (water)

N 46 ${ }^{\circ} 03.098^{\prime}$ W $122^{\circ} 37.369^{\prime} \pm 19$ ft

Elevation: $518 \mathrm{ft}$

Water temperature: approximately $14^{\circ} \mathrm{C}$

Specific conductance: $334 \mu \mathrm{S} / \mathrm{cm}$

This mineral spring is situated at the site of a former hotel built in 1906 for supposed healing properties of water. The mineral water was also bottled and sold for medicinal purposes. In late summer the spring has a slow seep rate. Spring water collects behind low concrete wall, which overflows and drains to the Kalama River, Washington. Water sample collected using siphon feed.

This mineral spring has been previously sampled for chemical analyses by Ivan Barnes and William Evans (April 1980), by Robert Mariner and William Evans (July 1980), by Ivan Barnes (November 1980), by Robert Mariner (July 1986), and by Rodney Caldwell (1993) as reported in Mariner and others (2006).

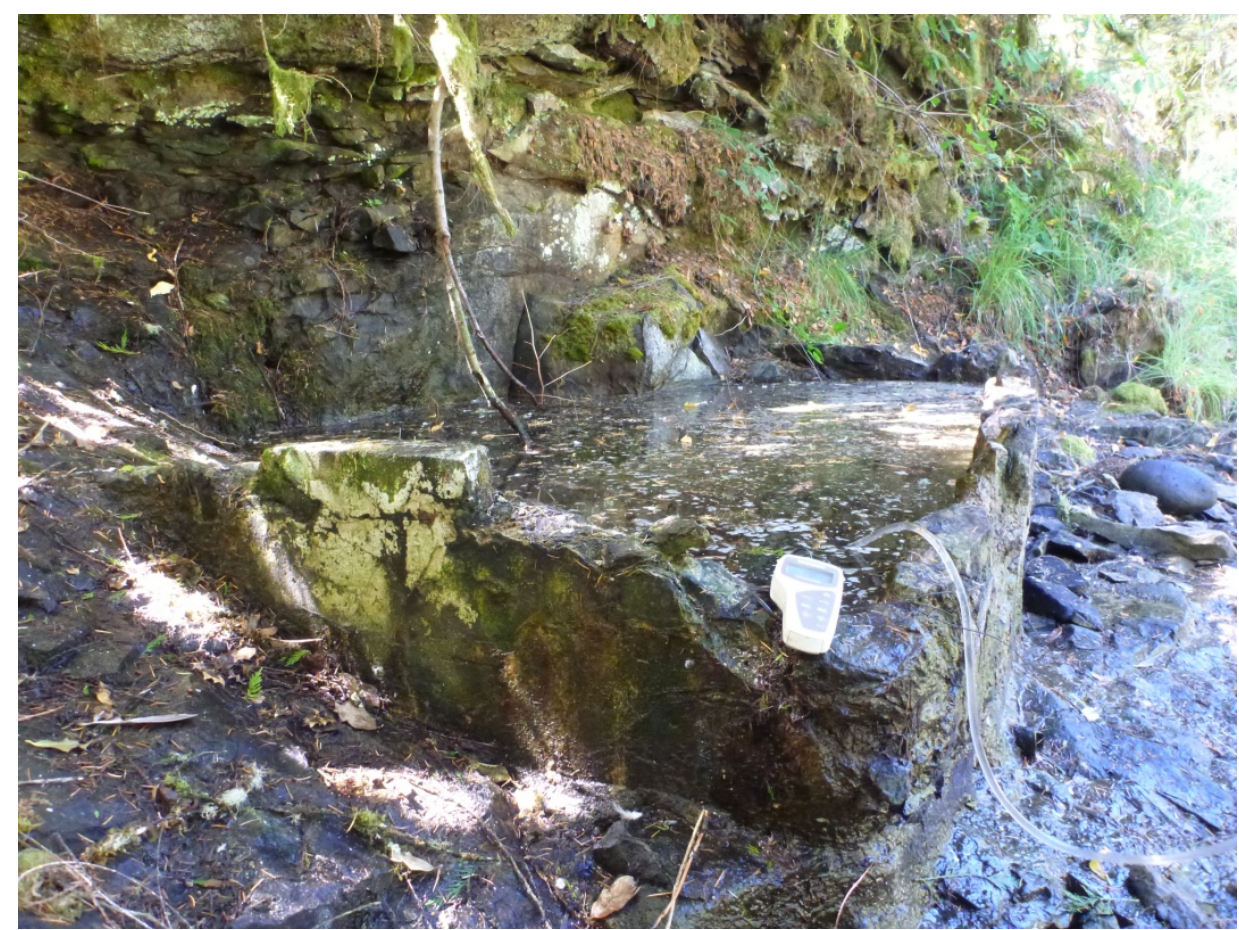




\section{Boswell Mineral Springs, Oregon}

Drain 7.5-minute topographic quadrangle map. Sampled August 2013. Juan de Fuca slab depth approximately $39 \mathrm{~km}$.

[Microsoft ${ }^{\mathbb{B}}$ Excel file of this table is available for download at http://pubs.usgs.gov/of/2014/1064/]

\begin{tabular}{|c|c|c|c|c|}
\hline Water sample & $\begin{array}{l}\mathrm{CH}_{4} \quad 1 \sigma \mathrm{err} \\
\left(\mathrm{ccSTP} / \mathrm{g} \times 10^{-3}\right) \\
\end{array}$ & $\begin{array}{ll}\mathrm{N}_{2} & 1 \sigma \mathrm{err} \\
\left(\mathrm{ccSTP} / \mathrm{g} \times 10^{-2}\right)\end{array}$ & $\begin{array}{lr}\mathrm{O}_{2} & 1 \sigma \mathrm{err} \\
\left(\mathrm{ccSTP} / \mathrm{g} \times 10^{-3}\right)\end{array}$ & $\begin{array}{ll}\mathrm{Ne} & 1 \sigma \mathrm{err} \\
\left(\mathrm{ccSTP} / \mathrm{g} \times 10^{-8}\right)\end{array}$ \\
\hline Boswell & 3.69 & 2.401 & $<0.02$ & 4.32 \\
\hline $\mathrm{ASW}$ at $20^{\circ} \mathrm{C}$ & & 1.17 & & 18.17 \\
\hline
\end{tabular}

\begin{tabular}{|c|c|c|c|c|c|}
\hline Water sample & $\begin{array}{l}\text { Ar } 1 \sigma \text { err } \\
\left(\mathrm{ccSTP} / \mathrm{g} \times 10^{-3}\right)\end{array}$ & $\begin{array}{l}\mathrm{Kr} \quad 1 \sigma \mathrm{err} \\
\left.\mathrm{ccSTP} / \mathrm{g} \times 10^{-8}\right)\end{array}$ & $\begin{array}{cc}\mathrm{Xe} & 1 \sigma \mathrm{err} \\
\left(\mathrm{ccSTP} / \mathrm{g} \times 10^{-8}\right)\end{array}$ & ${ }^{20} \mathrm{Ne} /{ }^{22} \mathrm{Ne}$ & $1 \sigma \mathrm{err}$ \\
\hline Boswell & 0.138 & 3.94 & 0.67 & 9.91 & \\
\hline ASW at $20^{\circ} \mathrm{C}$ & 0.306 & 6.84 & 0.93 & & \\
\hline
\end{tabular}

\begin{tabular}{c|cc|cc|cc}
\hline Water sample & ${ }^{40} \mathrm{Ar} /{ }^{36} \mathrm{Ar}$ & $1 \sigma \mathrm{err}$ & ${ }^{86} \mathrm{Kr} /{ }^{84} \mathrm{Kr}$ & $1 \sigma \mathrm{err}$ & ${ }^{130} \mathrm{Xe} /{ }^{132} \mathrm{Xe}$ & $1 \sigma \mathrm{err}$ \\
\hline $\begin{array}{l}\text { Boswell } \\
\text { ASW at } 20^{\circ} \mathrm{C}\end{array}$ & 302.3 & & 0.305 & & 0.153 & \\
\hline
\end{tabular}

Run notes:

1. ASW (Air Saturated Water) computed for listed temperature at an elevation of $500 \mathrm{ft}$.

2. Light noble gases stripped; $\mathrm{CH}_{4}$ noted; nitrogen rich sample.

3. $1 \sigma$ error for noble gases is approximately $1-2$ percent. 
20130821-01 PMc (water)

N 43 $38.424^{\prime}$ W $123^{\circ} 18.0669^{\prime}$

Elevation: $340 \mathrm{ft}$

Water temperature: approximately $10{ }^{\circ} \mathrm{C}$

This mineral spring is situated at the site of a former hunting lodge built in early 1900s (with train access only) for supposed healing properties of water. The mineral water was also bottled and sold for medicinal purposes. The spring water collects in a concrete cistern, which overflows into a field that drains to Elk Creek, Oregon. Water sample collected using siphon feed. [Note, another spring collects in a man-made pond at a higher elevation. Water from the pond is filtered by double osmosis for drinking purposes.]

No known water samples previously collected from this mineral spring.

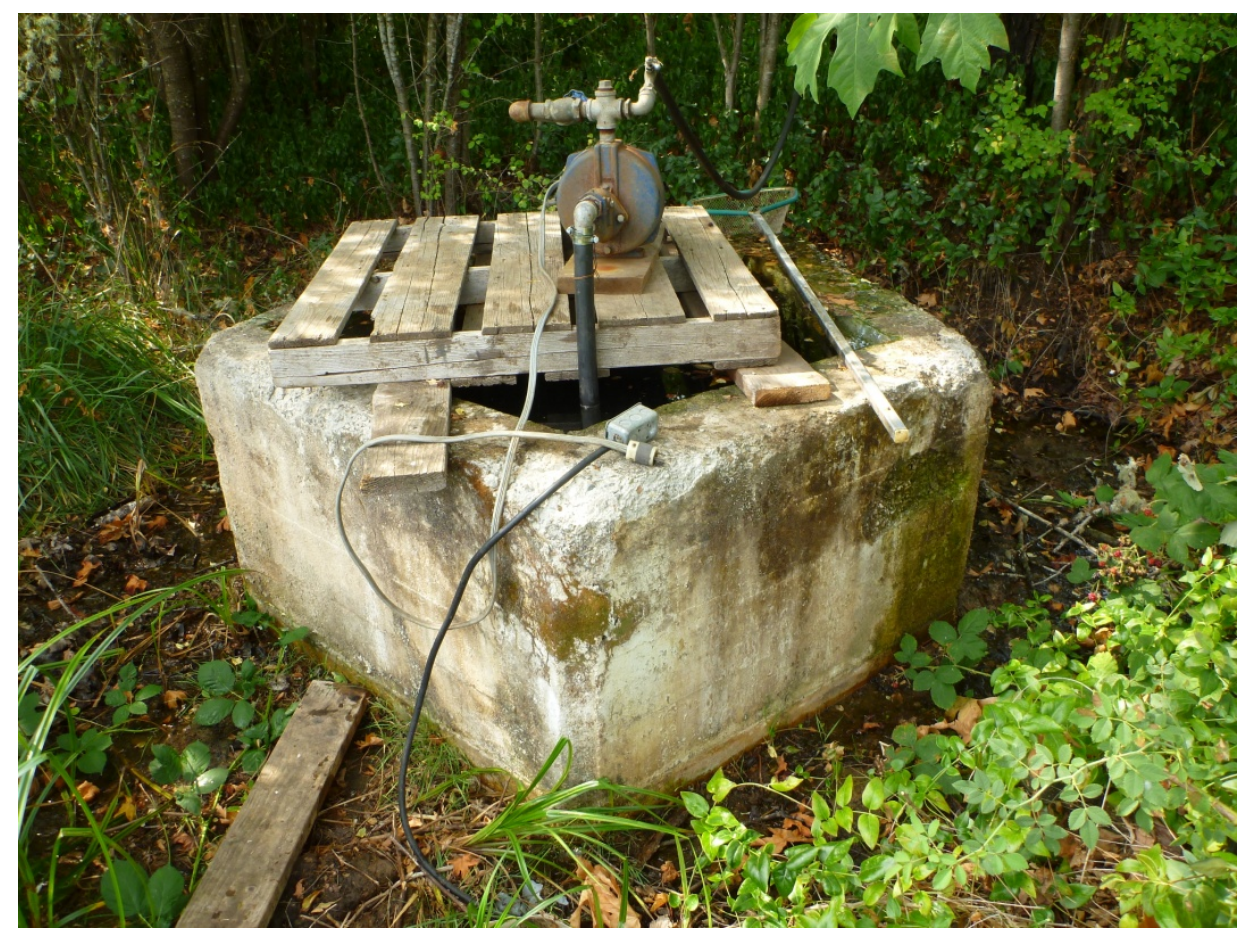




\section{Cascadia Soda Spring, Cascadia State Park, Oregon}

\section{Cascadia 7.5-minute topographic quadrangle map. Sampled August 2013. Juan de Fuca slab depth approximately $59 \mathrm{~km}$.}

[Microsoft ${ }^{\mathbb{R}}$ Excel file of this table is available for download at http://pubs.usgs.gov/of/2014/1064/]

\begin{tabular}{l|c|c|c|c}
\hline \multicolumn{1}{c|}{ Water sample } & $\begin{array}{c}\mathrm{CH}_{4} \quad 1 \sigma \mathrm{err} \\
\left(\mathrm{ccSTP} / \mathrm{g} \times 10^{-3}\right)\end{array}$ & $\begin{array}{c}\mathrm{N}_{2} \\
\left(\mathrm{ccSTP} / \mathrm{g} \times 10^{-3}\right)\end{array}$ & $\begin{array}{c}1 \sigma \mathrm{err} \\
\left(\mathrm{ccSTP} / \mathrm{g} \times 10^{-3}\right)\end{array}$ & $\begin{array}{c}\mathrm{Ne} \text { err } \\
\left(\mathrm{ccSTP} / \mathrm{g} \times 10^{-8}\right)\end{array}$ \\
\hline Cascadia & $<0.02$ & $<0.02$ & $<0.02$ & 1.94 \\
ASW at $20^{\circ} \mathrm{C}$ & & 1.17 & & 18.17 \\
\hline
\end{tabular}

\begin{tabular}{l|l|l|l|c|}
\hline \multicolumn{1}{c|}{ Water sample } & $\begin{array}{l}\mathrm{Ar} 1 \sigma \mathrm{err} \\
\left(\mathrm{ccSTP} / \mathrm{g} \times 10^{-3}\right)\end{array}$ & $\begin{array}{c}\mathrm{Kr} \quad 1 \sigma \mathrm{err} \\
\left(\mathrm{ccSTP} / \mathrm{g} \times 10^{-8}\right)\end{array}$ & $\begin{array}{c}\mathrm{Xe} \quad 1 \sigma \mathrm{err} \\
\left(\mathrm{ccSTP} / \mathrm{g} \times 10^{-8}\right)\end{array}$ & ${ }^{20 \mathrm{Ne} / 22 \mathrm{Ne}} 10 \mathrm{err}$ \\
\hline Cascadia & 0.041 & 1.11 & 0.2 & 9.99 \\
ASW at $20^{\circ} \mathrm{C}$ & 0.306 & 6.84 & 0.93 & \\
\hline
\end{tabular}

\begin{tabular}{c|cc|cc|cc}
\hline \multicolumn{1}{c|}{ Water sample } & ${ }^{40} \mathrm{Ar} /{ }^{36} \mathrm{Ar} \quad 1 \sigma \mathrm{err}$ & ${ }^{86} \mathrm{Kr} /{ }^{84} \mathrm{Kr}$ & $1 \sigma \mathrm{err}$ & ${ }^{130} \mathrm{Xe} /{ }^{132} \mathrm{Xe}$ & $1 \sigma \mathrm{err}$ \\
\hline Cascadia & 293.8 & 0.306 & & 0.153 & \\
ASW at $20^{\circ} \mathrm{C}$ & & & & & \\
\hline
\end{tabular}

Run notes:

1. ASW (Air Saturated Water) computed for listed temperature at an elevation of $500 \mathrm{ft}$.

2. Noble gases stripped by $\mathrm{CO}_{2}$; very large bubble of $\mathrm{CO}_{2}$ noted in bulk gas analysis. Note, we measured $\mathrm{CO}_{2}$ but do not report its value here because it represents a combination of dissolved $\mathrm{CO}_{2}$ and total inorganic carbon (DIC) that back reacts during sample extraction.

3. $1 \sigma$ error for noble gases is approximately $1-2$ percent. 
20130823-01 PMc (water)

N $44^{\circ} 23.957^{\prime}$ W $122^{\circ} 28.816$ '

Elevation: $921 \mathrm{ft}$

Water temperature: approximately $18^{\circ} \mathrm{C}$

This soda spring is situated at the site of a former hotel built in early 1900s for supposed healing properties of water. The spring is now contained within a state park. A park ranger opened the spring for access. The spring water collects in a metal cylinder, which seeps into Soda Creek, Oregon. We first attempted to collect a water sample using siphon feed, but the flow rate is extremely slow. Sample collected using syringe with a cloth to filter out fine red precipitate.

This soda spring has been previously sampled for chemical analyses by Robert Mariner (September 1985) as reported in Mariner and others (2006).

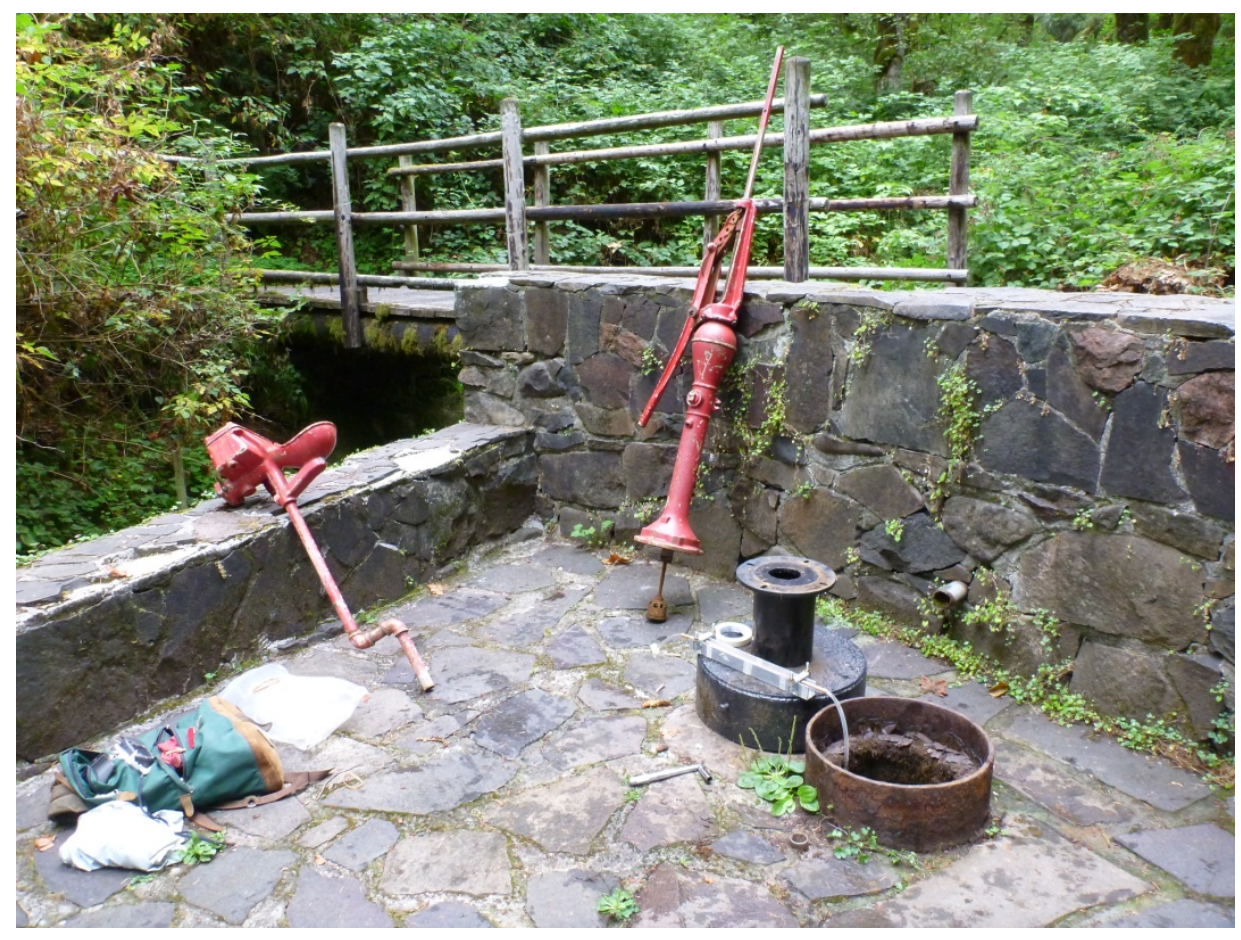




\section{Sodaville Mineral Spring, Sodaville Park, Oregon}

\section{Waterloo 7.5-minute topographic quadrangle map. Sampled August 2013. Juan de Fuca slab depth approximately $48 \mathrm{~km}$.}

[Microsoft ${ }^{\mathbb{R}}$ Excel file of this table is available for download at http://pubs.usgs.gov/of/2014/1064/]

\begin{tabular}{|c|c|c|c|c|}
\hline Water sample & $\begin{array}{l}\mathrm{CH}_{4} \quad 1 \sigma \mathrm{err} \\
\left(\mathrm{ccSTP} / \mathrm{g} \times 10^{-3}\right)\end{array}$ & $\begin{array}{lc}N_{2} & 1 \sigma \mathrm{err} \\
\left(\mathrm{ccSTP} / \mathrm{g} \times 10^{-3}\right) \\
\end{array}$ & $\begin{array}{lr}\mathrm{O}_{2} & 1 \sigma \mathrm{err} \\
\left(\mathrm{ccSTP} / \mathrm{g} \times 10^{-3}\right) \\
\end{array}$ & $\begin{array}{l}\mathrm{Ne} \\
\left(\mathrm{ccSTP} / \mathrm{g} \times 10^{-8}\right)\end{array}$ \\
\hline $\begin{array}{l}\text { Sodaville } \\
\text { ASW at } 20^{\circ} \mathrm{C}\end{array}$ & $<0.02$ & $\begin{array}{r}<0.02 \\
1.17\end{array}$ & $<0.02$ & $\begin{array}{l}16.91 \\
18.17\end{array}$ \\
\hline
\end{tabular}

\begin{tabular}{|c|c|c|c|c|c|}
\hline Water sample & $\begin{array}{l}\mathrm{Ar} \quad 1 \sigma \mathrm{err} \\
\left(\mathrm{ccSTP} / \mathrm{g} \times 10^{-3}\right)\end{array}$ & $\begin{array}{l}\mathrm{Kr} \\
\left(\mathrm{ccSTP} / \mathrm{g} \times 10^{-8}\right)\end{array}$ & $\begin{array}{l}\mathrm{Xe} \quad 1 \sigma \mathrm{err} \\
\left(\mathrm{ccSTP} / \mathrm{g} \times 10^{-8}\right)\end{array}$ & ${ }^{20} \mathrm{Ne} / 22 \mathrm{Ne}$ & $1 \sigma \mathrm{err}$ \\
\hline Sodaville & 0.287 & 6.64 & 0.93 & 9.82 & \\
\hline ASW at $20^{\circ} \mathrm{C}$ & 0.306 & 6.84 & 0.93 & & \\
\hline
\end{tabular}

\begin{tabular}{c|rc|cc|cc}
\hline \multicolumn{1}{c}{ Water sample } & ${ }^{40} \mathrm{Ar} /{ }^{66} \mathrm{Ar}$ & $1 \sigma \mathrm{err}$ & ${ }^{86} \mathrm{Kr} /{ }^{84} \mathrm{Kr}$ & $1 \sigma \mathrm{err}$ & ${ }^{130} \mathrm{Xe} /{ }^{132} \mathrm{Xe}$ & $1 \sigma \mathrm{err}$ \\
\hline $\begin{array}{l}\text { Sodaville } \\
\text { ASW at } 20^{\circ} \mathrm{C}\end{array}$ & 296.2 & & 0.304 & & 0.15 & \\
\hline
\end{tabular}

Run notes:

1. ASW (Air Saturated Water) computed for listed temperature at an elevation of $500 \mathrm{ft}$.

2. Noble gases stripped by $\mathrm{CO}_{2}$; very large bubble of $\mathrm{CO}_{2}$ noted in bulk gas analysis. Note, we measured $\mathrm{CO}_{2}$ but do not report its value here because it represents a combination of dissolved $\mathrm{CO}_{2}$ and total inorganic Carbon (DIC) that back reacts during sample extraction.

3. $1 \sigma$ error for noble gases is approximately $1-2$ percent. 
0130823-02 PMc (water)

N 44 29.057' W $122^{\circ} 52.126^{\prime}$

Elevation: $478 \mathrm{ft}$

Water temperature: approximately $16^{\circ} \mathrm{C}$

This mineral spring is situated at the site of a former hotel built in early 1900s for supposed healing properties of water. The spring is now contained within a town park. Spring water collects in a cistern, which overflows through metal pipe. Flow rate is about $1 \mathrm{~L} / \mathrm{min}$. Sample collected using siphon feed. Note, Sodaville water manager offered to open the cistern (within the adjacent shed), but the overflow pipe seemed adequate for sample collection.

This mineral spring has been previously sampled for chemical analyses by Robert Mariner and William Evans (September 1977) as reported in Mariner and others (2006).

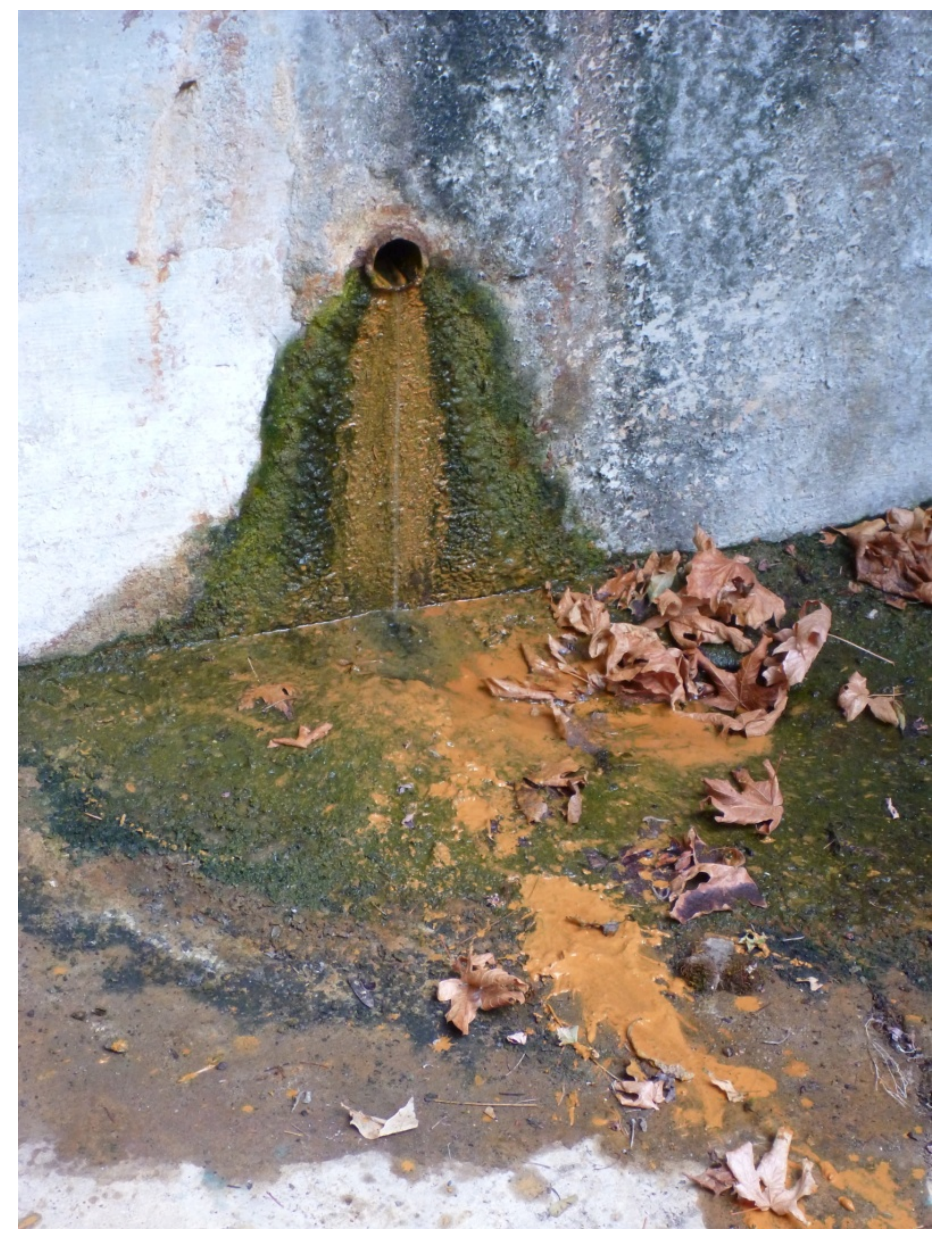




\section{Wilhoit Soda Spring, Wilhoit County Park, Oregon}

Wilhoit 7.5-minute topographic quadrangle map. Sampled August 2013. Juan de Fuca slab depth approximately $56 \mathrm{~km}$.

[Microsoft ${ }^{\circledR}$ Excel file of this table is available for download at http://pubs.usgs.gov/of/2014/1064/]

\begin{tabular}{|c|c|c|c|c|}
\hline Water sample & $\begin{array}{l}\mathrm{CH}_{4} \quad 1 \sigma \mathrm{err} \\
\left(\mathrm{ccSTP} / \mathrm{g} \times 10^{-3}\right)\end{array}$ & $\begin{array}{cc}\mathrm{N}_{2} & 1 \sigma \mathrm{err} \\
\left(\mathrm{ccSTP} / \mathrm{g} \times 10^{-3}\right)\end{array}$ & $\begin{array}{ll}\mathrm{O}_{2} & 1 \sigma \mathrm{err} \\
\left(\mathrm{ccSTP} / \mathrm{g} \times 10^{-3}\right)\end{array}$ & $\begin{array}{l}\mathrm{Ne} \\
\left(\mathrm{ccSTP} / \mathrm{g} \times 10^{-8}\right)\end{array}$ \\
\hline Sodaville & $<0.02$ & $<0.02$ & $<0.02$ & 0.87 \\
\hline ASW at $20^{\circ} \mathrm{C}$ & & 1.17 & & 18.17 \\
\hline
\end{tabular}

\begin{tabular}{|c|c|c|c|c|c|}
\hline Water sample & $\begin{array}{l}\mathrm{Ar} \quad 1 \sigma \mathrm{err} \\
\left(\mathrm{ccSTP} / \mathrm{g} \times 10^{-3}\right)\end{array}$ & $\begin{array}{l}\mathrm{Kr} \quad 1 \sigma \mathrm{err} \\
\left(\mathrm{ccSTP} / \mathrm{g} \times 10^{-8}\right)\end{array}$ & $\begin{array}{c}\mathrm{Xe} \quad 1 \sigma \mathrm{err} \\
\left(\mathrm{ccSTP} / \mathrm{g} \times 10^{-8}\right)\end{array}$ & ${ }^{20} \mathrm{Ne} / 22 \mathrm{Ne}$ & $1 \sigma \mathrm{err}$ \\
\hline Sodaville & 0.016 & 1.19 & 0.27 & 10.14 & \\
\hline $\mathrm{ASW}$ at $20^{\circ} \mathrm{C}$ & 0.306 & 6.84 & 0.93 & & \\
\hline
\end{tabular}

\begin{tabular}{l|cc|cc|cc}
\hline \multicolumn{1}{c}{ Water sample } & ${ }^{40} \mathrm{Ar} /{ }^{36} \mathrm{Ar} \quad 1 \sigma \mathrm{err}$ & ${ }^{86} \mathrm{Kr} /{ }^{84} \mathrm{Kr}$ & $1 \sigma \mathrm{err}$ & ${ }^{130} \mathrm{Xe} /{ }^{132} \mathrm{Xe}$ & $1 \sigma \mathrm{err}$ \\
\hline $\begin{array}{l}\text { Sodaville } \\
\text { ASW at } 20^{\circ} \mathrm{C}\end{array}$ & 301.8 & 0.308 & & 0.145 & \\
\hline
\end{tabular}

Run notes:

1. ASW (Air Saturated Water) computed for listed temperature at an elevation of $500 \mathrm{ft}$.

2. Noble gases stripped by $\mathrm{CO}_{2}$; very large bubble of $\mathrm{CO}_{2}$ noted in bulk gas analysis. Note, we measured $\mathrm{CO}_{2}$ but do not report its value here because it represents a combination of dissolved $\mathrm{CO}_{2}$ and total inorganic carbon (DIC) that back reacts during sample extraction.

3. $1 \sigma$ error for noble gases is approximately $1-2$ percent. 
20130823-03 PMc (water)

$\mathrm{N} 45^{\circ}$ 03.141' W $122^{\circ} 34.094$ '

Elevation: $728 \mathrm{ft}$

Water temperature: approximately $18{ }^{\circ} \mathrm{C}$

This soda spring is situated at the site of a former hotel built in early 1900s for supposed healing properties of water. People still collect spring water to drink as it is now contained within a county park. Soda water collects in a metal cylinder, which overflows into Rock Creek, Oregon. Moderate flow rate. This spring releases large gas bubbles every few minutes. Collected using siphon feed. Some gas in water sample.

Note, this park also contains a 'sulphur' spring, with water obtained using an old-fashioned hand pump. The pump, however, was broken in 2013. No seepage observed between pump and stream.

This mineral spring has been previously sampled for chemical analyses by Robert Mariner and William Evans (September 1977) as reported in Mariner and others (2006).

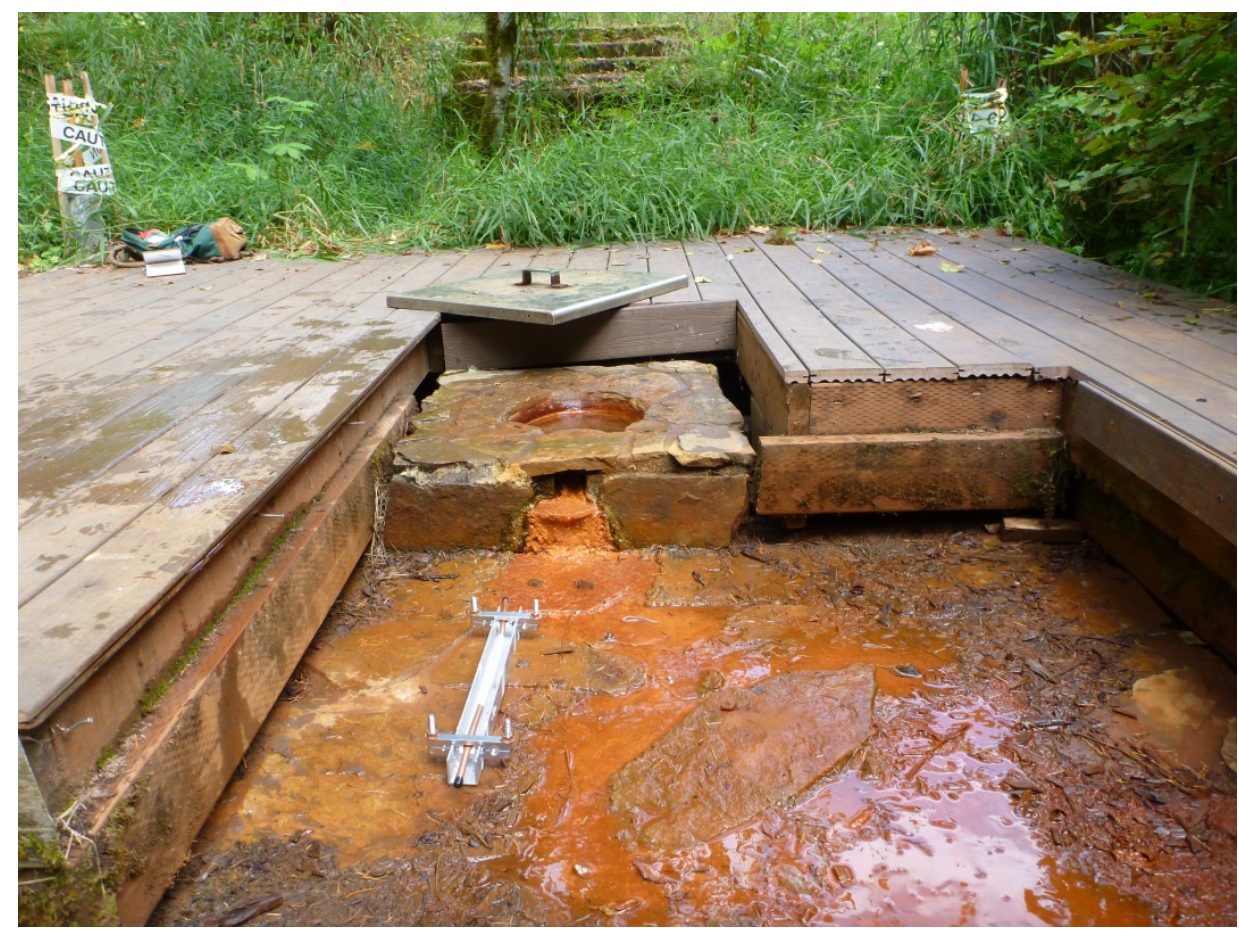




\section{Acknowledgments}

We would like to thank the Olympic National Park in Washington and the Cascadia, Sodaville, and Wilhoit Parks in Oregon for providing access to mineral and soda springs within their parks. We would also like to thank those private landowners who permitted access to mineral springs on their land in Washington and Oregon, namely, William Rountree and his neighbors, John and Martha Oyala, and Johna Cook, as well as the Jackson Prairie Natural Gas Storage Facility, Washington. We also would like to thank Robert Mariner and William Evans for assistance in locating remote mineral springs. Review comments by William Evans and Patrick Dobson improved this contribution.

\section{References Cited}

Benson, B.B., and Krause, D., 1976, Empirical laws for dilute aqueous solutions of non-polar gases: Journal of Chemical Physics, v. 64, p. 689-709.

Mariner, R.H., Venezky, D.Y., and Hurwitz, S., 2006, Chemical and isotopic database of water and gas from hydrothermal systems with an emphasis for the western United States: U.S. Geological Survey Data Series 169, http://pubs.usgs.gov/ds/2005/169/.

McCrory, P.A., Blair, J.L., Waldhauser, Felix, and Oppenheimer, D.H., 2012, Juan de Fuca slab geometry and its relation to Wadati-Benioff zone seismicity: Journal of Geophysical Research, v. 117, n. B09306, 23 p., doi:10.1029/2012JB009407.

McCrory, P.A., Hyndman, R.D., and Blair, J.L., 2014, Relationship between the Cascadia forearc mantle wedge, non-volcanic tremor, and the downdip limit of seismogenic rupture: Geochemistry, Geophysics, Geosystems, v. 15, doi:10.1002/2013GC005144.

Weiss, R.F., 1970, The solubility of nitrogen, oxygen and argon in water and seawater: Deep-Sea Research, v. 17, p. 721-735.

Weiss, R.F., 1971, Solubility of helium and neon in water and seawater: Journal of Chemical and Engineering Data, v. 16, p. 235-241.

Weiss, R.F., and Kyser, T.K., 1970, Solubility of krypton in water and seawater: Journal of Chemical and Engineering Data, v. 16, p. 235-241, doi: 10.1021/je60076a014.

Wilson, D.S., 2002, The Juan de Fuca plate and slab: Isochron structure and Cenozoic plate motions: U.S. Geological Survey Open-File Report 02-328, p. 9-12. 